\title{
Biophysical Basis of Thermometry Limitations to Control COVID-19 are Overcome at Transmissive Skin Overlying Brain-eyelid Thermal Tunnels
}

\author{
Marcio Marc Abreu ${ }^{1,2}$, Ricardo L Smith ${ }^{3}$, Trevor M Banack ${ }^{1}$, Anna L Clebone ${ }^{1}$, Ala S \\ Haddadin $^{1}$, Tyler J Silverman ${ }^{1}$, Feng Dai ${ }^{4}$, and David G Silverman ${ }^{1,5}$ \\ ${ }^{1}$ Department of Anesthesiology, Yale University School of Medicine \\ ${ }^{2}$ Department of Ophthalmology and Visual Science, Yale University School of Medicine \\ ${ }^{3}$ Department of Morphology and Genetics, Paulista School of Medicine, Federal University \\ of São Paulo \\ ${ }^{4}$ Yale Center for Analytical Sciences, Yale School of Public Health, Yale University \\ ${ }^{5}$ John B. Pierce Foundation Laboratory, Yale University
}

October 28, 2020

\begin{abstract}
Essential to management of pandemics, noninvasive thermometry has been hampered by inaccuracies and inconsistencies, due to measurements on low thermal conductivity $(k)$ surfaces we show here, which have precluded effective assessment of febrile states for centuries. Discovery of brain-eyelid thermal tunnels (BTT) identified undisturbed thermal transmission from brain. We here report a series of integrated anatomic, histologic, thermal emissive, thermal physiologic and thermometric studies designed to identify, characterize and overcome the biophysical limitations of surface thermometry as currently used worldwide to control COVID-19, which primarily relies on non-contact forehead thermometry. However, the forehead has thick and variable dermis $(\sim 2000 \mu \mathrm{m}$ to $\sim 2500 \mu \mathrm{m})$ and fat layers $(\sim 1100 \mu \mathrm{m}$ to $\sim 2800 \mu \mathrm{m})$ resulting in low- $k$ insulation equivalent to wood. During thermal emission, the highest skin temperature at forehead, present in only $\sim 3.1 \%$ of forehead, averaged $1.07 \pm 0.49^{\circ} \mathrm{C}(\mathrm{mean} \pm \mathrm{SD})$ less than over BTT $\left(\mathrm{p}=0.008\right.$, two-tailed paired t-test). Thermometric studies (without correction factor) revealed $1.97^{\circ} \mathrm{C}$ higher temperature at $\mathrm{BTT}$ site $\left(\mathrm{BTT}^{\circ}\right)$ than forehead. Facial fanning caused $3.5^{\circ} \mathrm{C}$ reduction in forehead temperature, which do not compromise BTT site, attributable to impact on non-BTT surface sites by highly variable surface vasculature. Cerebral hemisphere dominance studies uncovered higher $\mathrm{BTT}^{\circ}$ in the dominant side $\left(\mathrm{P}<0.001\right.$ for $0.14^{\circ} \mathrm{C}$ difference). Like $\mathrm{BTT}$, superior palpebral vein region has thin dermis $(\sim 900 \mu \mathrm{m})$ and is fat-free, however, BTT has higher intensity light emission due to heat transfer from underlying brain tunnel. Findings bring a new dimension for combating COVID-19 that is aligned with physics and biology, and without any cost whatsoever and undue burden, current worldwide suboptimal low- $k$ thermometry can be immediately and conveniently enhanced by brain-enabled thermo-physical based high- $k$ signal conversion, which can be easily implemented by any country, community or individual in the world.
\end{abstract}

\section{Hosted file}

Authorea_Biophysics-Thermometry-Limit_Control-COVID-19_via-BTT_TLY_Abreu et al_Yale Univ_0ct-2020F2_Sub available at https://authorea.com/users/364278/articles/489287-biophysical-basis-ofthermometry-limitations-to-control-covid-19-are-overcome-at-transmissive-skin-overlyingbrain-eyelid-thermal-tunnels

Rich media available at https://vimeo.com/462269350

Rich media available at https://vimeo.com/462269319 
Rich media available at https://vimeo.com/462269295 